\title{
nCPAP Improves the Quality of Life of Siblings with Mandibuloacral Dysplasia
}

\author{
Kumi Kato-Nishimura ${ }^{1}$, Ikuko Mohri, ${ }^{1,2}$, Shin Nabatame ${ }^{3}$, Motohiro Akagi ${ }^{3}$, Norio Sakai ${ }^{3}$, \\ Yoko Miyoshi $^{3}$, Keiichi Ozono ${ }^{3}$, Naoko Tachibana ${ }^{4}$ and Masako Taniike*,1,2
}

\begin{abstract}
${ }^{I}$ Department of Mental Health and Environmental Effects Research, Molecular Research Center For Children's Mental Development, Osaka University Graduate School of Medicine, Osaka, Japan; ${ }^{2}$ Department of Child Development, United Graduate School of Child Development, Osaka University; ${ }^{3}$ Department of Developmental Medicine (Pediatrics), Osaka University Graduate School of Medicine, Osaka, Japan; ${ }^{4}$ Center for Sleep-Related Disorders, Kansai Electric Power Company Hospital, Osaka, Japan
\end{abstract}

\begin{abstract}
Mandibuloacral dysplasia (MAD; MIM 248370, 608612) is a rare progeroid syndrome with autosomal recessive inheritance. It is characterized by mandibular hypoplasia, acroosteolysis, delayed closure of the cranial sutures, skin atrophy with mottled hyperpigmentation, stiff joints, and growth retardation. We here report Japanese female siblings with a severe MAD phenotype. Because of extreme micrognathia and small mouth and nostril, obstructive sleep apnea syndrome (OSAS) was observed in both sisters and was especially life-threatening in the younger sister. Nasal continuous positive airway pressure (nCPAP), which seemed to be only one therapeutic choice for these sisters since impaired bone healing made oral surgical approach including maxillomandibular advancement surgery inapplicable to these sisters, successfully alleviated OSAS in both sisters. Since the initiation of nCPAP, the younger sister has gained weight constantly and her developmental milestones have been steadily achieved. We conclude that possible life threatening sleepdisordered breathing in the patients with progeroid syndromes should be properly managed.
\end{abstract}

Keywords: Progeroid syndrome, OSAS, nCPAP, micrognathia, ZMPSTE24.

\section{INTRODUCTION}

Mandibuloacral dysplasia (MAD; MIM 248370, 608612) is a rare progeroid syndrome with characteristic manifestations including mandibular hypoplasia, acroosteolysis, delayed closure of the cranial sutures, cutaneous atrophy, lipodystrophy, joint contractures, and growth retardation [1]. Symptoms appear around four years of age in most MAD patients. In MAD patients, mutations in lamin $\mathrm{A} / \mathrm{C}$ gene [2] or zinc metalloproteinase gene [3] have been reported, which is involved in prelamin A processing. Agarwal et al. reported a 7-yr-old girl with MAD having LMNA mutation belonging to a consanguineous pedigree [4]. These mutations lead to the accumulation of farnesylated form of prelamin A and disruption of the structural scaffolding for the cell nucleus [3]. Using mouse model, the investigation of pathophysiology and therapeutic trials has been just started [5], however, at the moment, appropriate supportive care is crucial for improving quality of life (QOL) of MAD patients.

Although facial features of MAD are risk factors for obstructive sleep apnea syndrome (OSAS), there have been no reports documenting co-morbidity and management of OSAS in MAD. Here, we report two Japanese female siblings with a severe form of MAD. They are the first Japanese

*Address correspondence to this author at the Department of Mental Health and Environmental Effects Research, The Molecular Research Center for Children's Mental Development, Osaka University Graduate School of Medicine, 2-2 Yamada-oka, Suita, Osaka 565-0871, Japan; Tel: +81-6-8793932; Fax: +81-6-879-3939; E-mail: masako@kokoro.med.osaka-u.ac.jp cases and in both of them, potentially life-threatening OSAS was successfully treated by nasal continuous positive airway pressure (nCPAP) intervention.

\section{CASE REPORT}

Cases 1 and 2 were born to healthy and unconsanguineous parents. Mutations of zinc metalloproteinase gene were found in both cases [6]. Short stature and characteristic scleroderma were noted at birth, and most features of MAD became full blown before 6 months of age in both cases. Skeletal X-ray identifying multiple lambdoidal wormian bones and osteolysis of the distal phalanges confirmed the diagnosis of MAD.

Their mother noticed the elder sister (Case 1) snored when she was 6 months old, and witnessed cessation of breathing during sleep at the age of 3 years. At 3 years of age, Case 1 exhibited midface hypoplasia, severe micrognathia and small nostrils (Fig. 1A). Her blood pressure, 106 / $70 \mathrm{mmHg}$, was mildly high for her age of three. Electrocardiogram, echocardiogram and hematological/blood chemical analyses were normal. Although her mental development was remarkably good, she was hyperactive and extremely stubborn. Sagittal images of cranial magnetic resonance (MR) indicated severe micrognathia as well as adenoid hypertrophy (Fig. 1B). Mandibular fracture resulting from crowded teeth (Fig. 1C) was discovered by chance, and bone healing was severely delayed.

A standard overnight polysomnography (PSG) was performed. We employed a thermister to measure air flow and apnea was defined as cessation of breathing for more than 

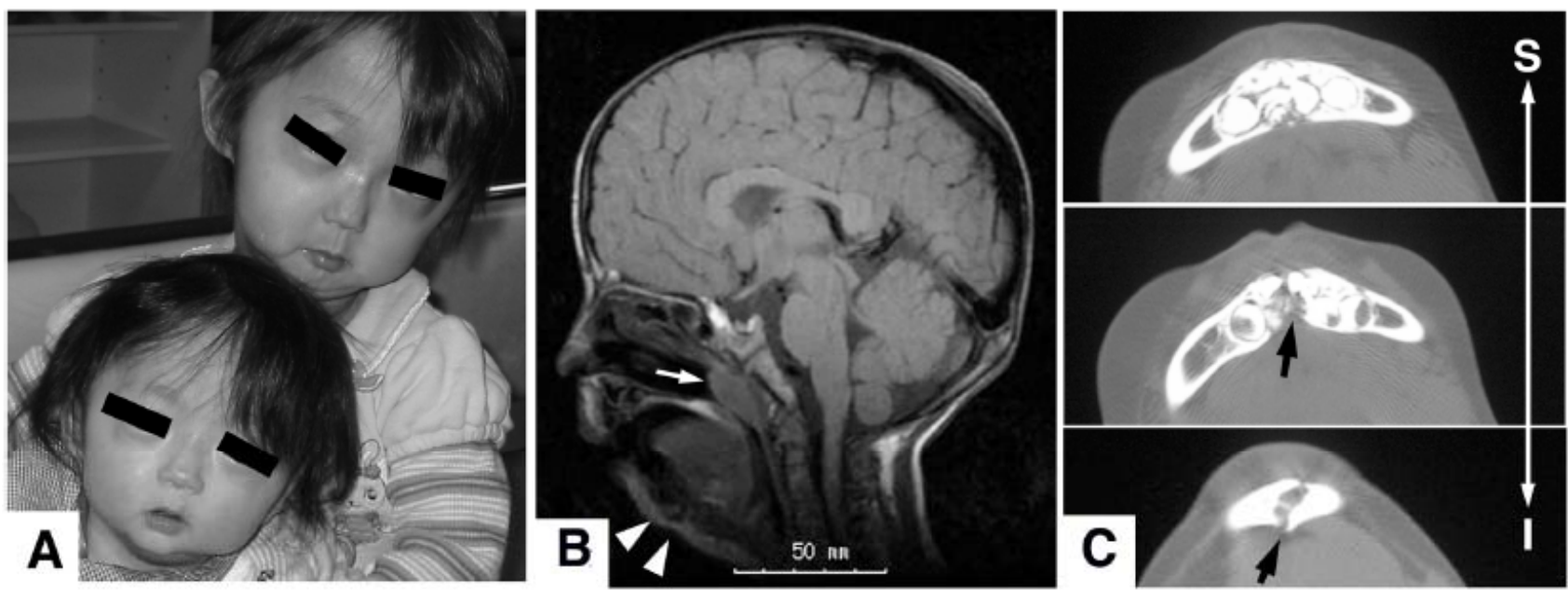

Fig. (1). A: Photographs of sisters with MAD. Note their prominent eyes, midfacial hypoplasia, small nostril and mouth, and micrognathia. B: A sagittal image of cranial MR of the elder sister. Hypertrophied adenoid occludes upper airway (an arrow). Note the hypoplastic mandible (arrowheads). C: Computed tomography of the mandibular bone of the elder sister. S-I indicates a superior-inferior direction. Note the overcrowded teeth (the top panel) leading to the bone fracture (arrows in the center and the bottom panel).

two breaths in compliance with the American Thoracic Society criteria consensus for children [7]. Upon confirming apnea-hypopnea index (AHI) of 26.6 and desaturation index (DI: the number of respiratory events with oxygen desaturation) $\geq 3 \%$ (Table 1), nCPAP was initiated with a belt-type headgear and Comfort nasal mask (ML size; Respironics, Murrysville, PA) as a full-face mask. Snoring and obvious apneas disappeared at the pressure of $12 \mathrm{cmH}_{2} \mathrm{O}$. nCPAP calibration was done on two consecutive nights with the first night for a diagnostic PSG and the second one for nCPAP calibration. AHI, DI, and arousal index associated with respiratory events (ArI) were remarkably decreased on nCPAP treatment (Table 1). Her blood pressure was normalized to $80 / 46 \mathrm{mmHg}$. After the initiation of nCPAP, her hyperactivity was improved and she became to be able to sit down for 1 hour and became a biddable child. Therefore, despite the lack of the objective evidence, we can assume that sleep disturbance lead to her behavioral problems before nCPAP. Subsequent adenotonsillectomy was performed at the age of 5 years, yet, nCPAP treatment has remained indispensable.

As for the younger sister (Case 2), the features of MAD were already presented at birth. Since she showed severe respiratory distress, feeding difficulty and failure to thrive, she was admitted to our hospital when she was 6 months old. Remarkable respiratory effort and frequent obstructive apnea were observed concomitant with oxygen desaturation below $80 \%$ and inability to maintain sleep for longer than one hour at a stretch. She was too hypotonic to hold her head up and seldom smiled. No tonsillar or adenoid hypertrophy was present.

After an episode of severe aspiration pneumonia, nCPAP using Ultramirage (Shallow, ResMed) mask with a belt-type headgear was urgently initiated. As we used this mask as an full face mask, no significant leakage was identified. nCPAP resulted in control of snoring and episodes of $\mathrm{SpO}_{2}<90 \%$ at the pressure of $8 \mathrm{cmH}_{2} \mathrm{O}$. She slept for 7-8 hours in one stretch and daytime respiratory distress completely disappeared. She had a severe failure to thrive; she was $4.1 \mathrm{~kg}$ at 6month-old and her body weight gain is $-1 \mathrm{~g} /$ day during 1 month just before initiating nCPAP. After nCPAP, her body weight increase $28 \mathrm{~g} /$ day.

Fitting of the masks was checked every month and most fitted one was selected according to the growth of the facial bones. Mandibular fracture caused by crowded teeth was also discovered in the regular follow-up.

\section{DISCUSSION}

The cases presented here suffered from severe MAD. Review of their clinical courses disclosed that treatment of OSAS was crucial for MAD management. It has been reported that major risk factors for OSAS in children include hypertrophy of the tonsils and adenoids, neuromuscular diseases, obesity, and genetic craniofacial syndromes [7]. Midface hypoplasia, small nostrils, and micrognathia in our cases comprise risk factors for OSAS [8], however, to the best of our knowledge, this is the first report documenting the management of OSAS in progeroid syndromes including MAD. Since one neonatal MAD case was reported to die from "apnoeic episodes" [9], it is plausible that the presence of sleepdisordered breathing has been overlooked in progeroid syndromes.

nCPAP has been documented to be effective in ameliorating pediatric OSAS, and especially in the cases when adenotonsillectomy fails to mitigate the condition, nCPAP is the only choice of treatment. In our cases, nCPAP treatment resulted in behavioral improvement, normalization of the blood pressure, elimination of life-threatening respiratory distress and achievement of physical and psychomotor development. For these sisters, nCPAP was the only viable choice, since impaired bone healing (Fig. 1C) made an oral surgical approach including maxillomandibular advancement inapplicable [10]. Finally, efficient treatment by nCPAP might decrease the risk of the future cardiopulmonary complications associated with OSAS [11], and lengthen the life expectancy of the potentially short-lived patients with progeroid syndromes. We are now following up more than 6 years and now they are 11 and 8 year-old, and our cases encouraged pediatricians to look for the presence of sleep- 
Table 1. Changes in Sleep and Respiratory Parameters Before and After nCPAP in Cases 1 and 2

\begin{tabular}{|c|c|c|}
\hline Case 1 & Baseline & Treatment \\
\hline Total sleep time (min) & 397.0 & 563.5 \\
\hline Sleep efficiency $(\%)$ & 87.9 & 91.6 \\
\hline$\%$ WASO & 12.1 & 8.5 \\
\hline$\%$ Stage 1 & 5.5 & 5.9 \\
\hline$\%$ Stage 2 & 28.6 & 23.5 \\
\hline$\%$ Stage 3 & 12.6 & 14.5 \\
\hline$\%$ Stage 4 & 28.6 & 23.6 \\
\hline$\%$ REM & 12.6 & 24.0 \\
\hline Apnea hypopnea index ( /h) & 26.6 & 2.7 \\
\hline in REM sleep & 51.6 & 2.0 \\
\hline in NREM sleep & 22.4 & 2.9 \\
\hline Apnea index $(/ \mathrm{h})$ & 11.0 & 2.2 \\
\hline $3 \%$ Desaturation index $(/ \mathrm{h})$ & 24.9 & 6.0 \\
\hline$\%$ time $\mathrm{SpO}_{2}<90 \%$ & 2.3 & 0.1 \\
\hline Arousal index $(/ \mathrm{h})$ & 11.9 & 7.3 \\
\hline spontaneous & 7.3 & 7.3 \\
\hline associated with respiratory event & 4.6 & 0.0 \\
\hline in REM sleep & 4.2 & 2.0 \\
\hline in NREM sleep & 13.2 & 2.9 \\
\hline Case 2 & Baseline & Treatment \\
\hline 3\% Desaturation index $(/ \mathrm{h})$ & $98.9 \pm 15.7$ & $15.6 \pm 3.6$ \\
\hline$\%$ time $\mathrm{SpO}_{2}<90 \%$ & $14.9 \pm 5.1$ & $0.6 \pm 0.1$ \\
\hline
\end{tabular}

disordered breathing in the patients with progeroid syndromes.

\section{ACKNOWLEDGEMENT}

We thank for Mr. Ryosaku Ushiro, Koike Medical Co. Ltd., for valuable assistance and technical support.

\section{ABBREVIATIONS}

OSAS = obstructive sleep apnea syndrome

nCPAP = Nasal continuous positive airway pressure

MAD = Mandibuloacral dysplasia

PSG = polysomnography

\section{REFERENCE}

[1] Schrander-Stumpel C, Spaepen A, Fryns JP, Dumon J. A severe case of mandibuloacral dysplasia in a girl. Am J Med Genet 1992; 43(5): 877-81.

[2] Novelli G, Muchir A, Sangiuolo F, et al. Mandibuloacral dysplasia is caused by a mutation in LMNA-encoding lamin A/C. Am J Hum Genet 2002; 71(2): 426-31.
[3] Agarwal AK, Fryns JP, Auchus RJ, Garg A. Zinc metalloproteinase, ZMPSTE24, is mutated in mandibuloacral dysplasia. Hum Mol Genet 2003; 12(16): 1995-2001.

[4] Agarwal AK, Kazachkova I, Ten S, Garg A. Severe mandibuloacral dysplasia-associated lipodystrophy and progeria in a young girl with a novel homozygous Arg527Cys LMNA mutation. J Clin Endocrinol Metab 2008; (12): 4617-23.

[5] Fong LG, Frost D, Meta M, Qiao X, Yang SH, Coffinier C, et al. A protein farnesyltransferase inhibitor ameliorates disease in a mouse model of progeria. Science 2006; 311: 1621-3.

[6] Miyoshi Y, Akagi M, Agarwal A, et al. Severe mandibuloacral dysplasia caused by novel compound heterozygous ZMPSTE24 mutations in two japanese siblings. Clin Genet 2008;in press.

[7] Zen TSA. Standards and indications for cardiopulmonary sleep studies in children. American Thoracic Society. . Am J Respir Crit Care Med 1996; 153: 866-78.

[8] Handler SD. Upper airway obstruction in craniofacial anomalies: diagnosis and management. Birth Defects Orig Artic Ser 1985; 21(2): $15-31$

[9] Seftel MD, Wright CA, Po PL, de Ravel TJ. Lethal neonatal mandibuloacral dysplasia. Am J Med Genet 1996; 66(1): 52-4.

[10] Prinsell JR. Maxillomandibular advancement surgery for obstructive sleep apnea syndrome. J Am Dent Assoc 2002; 133(11): 1489-97.

[11] Lipton AJ, Gozal D. Treatment of obstructive sleep apnea in children: do we really know how? Sleep Med Rev 2003; 1: 61-80.

(C) Kato-Nishimura et al.; Licensee Bentham Open.

This is an open access article licensed under the terms of the Creative Commons Attribution Non-Commercial License (http://creativecommons.org/licenses/by-nc/3.0/) which permits unrestricted, non-commercial use, distribution and reproduction in any medium, provided the work is properly cited. 